Cahiers d'études italiennes

\title{
Les figures de la séduction dans les dessins de Poema a fumetti de Dino Buzzati
}

Muriel Badet

\section{OpenEdition}

\section{Journals}

Édition électronique

URL : http://journals.openedition.org/cei/843

DOI : $10.4000 /$ cei.843

ISSN : 2260-779X

Éditeur

UGA Éditions/Université Grenoble Alpes

Édition imprimée

Date de publication : 15 septembre 2006

Pagination : 255-266

ISBN : 978-2-84310-086-4

ISSN : 1770-9571

\section{Référence électronique}

Muriel Badet, «Les figures de la séduction dans les dessins de Poema a fumetti de Dino Buzzati », Cahiers d'études italiennes [En ligne], 5 | 2006, mis en ligne le 15 mars 2008, consulté le 27 mars 2021 URL : http://journals.openedition.org/cei/843 ; DOI : https://doi.org/10.4000/cei.843 


\title{
LES FIGURES DE LA SÉDUCTION DANS LES DESSINS DE POEMA A FUMETTI DE DINO BUZZATI
}

\author{
Muriel Badet \\ EHESS, Paris
}

Picasso à Buzzati ${ }^{1}$ :

- Si vous ne voulez pas avoir une indigestion de poison cessez de peindre. Vous, vous êtes écrivain. Le monde - je veux dire votre monde, qu'il soit petit ou grand - a de la considération pour vous en tant qu'écrivain.

Et Picasso de poursuivre:

- ... sur le plan pictural... Oui, oui, une certaine intelligence dans l'invention. Mais en ce qui concerne le métier! de grâce! ici nous sommes en plein dans la BD! Un chromatisme sommaire. C'est inutile, lorsque les bases font défaut (un petit rire) Littérature! Littérature! la peinture, c'est autre chose!

Comme pour répondre à l'entretien avec Picasso, Buzzati aurait déclaré, non sans provocation ${ }^{2}$ :

La peinture n'est pas pour moi un hobby: c'est au contraire l'écriture qui en est un. De toute manière, que je peigne ou que j'écrive, je poursuis toujours le même but qui, après tout, est simplement de raconter des histoires.

Dino Buzzati est principalement connu comme écrivain et comme chroniqueur au Corriere della Sera. En novembre 1959, il en devient le critique d'art. Il est aussi peintre. Ses premières œuvres, des portraits de Beatrice Giacometti, la jeune fille qu'il aime, ou des autoportraits, datent des années 1924-1928 3 . Tout au long de sa vie, Buzzati continue à peindre et

1. Cité par Donat-Cattin A., «Le peintre», Cahiers Dino Buzzati, n², Paris, Laffont, 1978, p. 219.

2. Ibid, p. 216.

3. Buzzati D., Romantica, 1924. Encre sur papier, 55 x 37, coll. privée; Il Lampione, 1926. Tempera sur papier, 41 x 43, coll. privée; Primo amore, 1930. Encre sur papier, 45 x 36, coll. privée. Toutes les dimensions des œuvres sont en centimètres. L'indication de la technique huile sur toile se simplifie en $H T$. 
à dessiner. En 1945, il écrit et illustre La Famosa invasione degli orsi in Sicilia; en 1958 se tient sa première exposition personnelle à la galerie Re Magi à Milan; en 1959, il réalise pour la Scala les décors pour Jeu de cartes de Stravinsky.

En automne 1968, Buzzati propose à l'éditeur Arnoldo Mondadori La Dolce morte, manuscrit qui deviendra quelques mois plus tard Poema a fumetti (Poème-Bulles pour la traduction française). Poema a fumetti ${ }^{4}$ se présente sous forme d'un livre d'images où se mêlent, sur une même page, texte et dessin en couleur. Ouvrage singulier, difficilement identifiable, ni un roman, ni vraiment un conte fantastique ou un récit mythologique illustré, encore moins une bande dessinée, Poema a fumetti ne peut en rien être considéré comme un hasard: il est même la concrétisation du travail pictural de Buzzati.

Ce texte fait du désir et de la séduction le sujet principal de sa narration. Un homme, Orfi, souhaite ramener à la vie la femme qu'il aime, Eura, et pour cela il descend la chercher dans le monde souterrain et urbain de l'au-delà. Dans cette histoire-prétexte, transposition à l'ère contemporaine de la descente aux Enfers d'Orphée, parti quérir Eurydice, la séduction passe des mots aux images, de la citation à l'invention, accentuant la singularité des situations, les orientant vers un sentiment d'inquiétante étrangeté où le déjà vu, le déjà lu voisinent avec l'univers personnel et fantastique de Buzzati.

Au travers des dessins, présences prépondérantes du livre, au moins quatre figures de la séduction apparaissent: le chanteur, l'image de la disponibilité des femmes, le travail de la citation et le personnage du séducteur.

\section{Le chanteur}

Dans la mythologie, la voix enchanteresse d'Orphée séduit tous ceux qui l'écoutent. Le son de sa lyre charme animaux, arbres et rochers et pacifie la nature $^{5}$. Ce chant permet au héros de franchir le seuil de l'Hadès: il fait

4. Poema a fumetti, Milan, A. Mondadori Editore, 1969, est composé de 224 planches, au départ dessinées à l'encre de chine sur un format de 30 x $40 \mathrm{~cm}$ puis colorées à la demande de l'éditeur. Publié en 1969, Buzzati obtint le prix Premio Paese Sera. L'édition consultée: Milan, Oscar Mondadori, 1991.

5. Ovide, Les Métamorphoses, Paris, les Belles lettres, 1991, X; Apollonios de Rhodes, Les Argonautiques, Paris, les Belles lettres, 1976, 1, 26-29: «On conte qu’il avait charmé dans les montagnes les durs rochers et le cours des fleuves par la musique de ses chants." 
taire Cerbère et fléchit les divinités infernales qui l'autorisent à reprendre Eurydice. L'étymologie de son nom précise l'être dans le nom et la dualité qui l'habite: Orphée est voix et mélopée mais il est aussi obscurité, celui qui accède aux Ténèbres. Il est le premier, avant Hercule et Thésée, à tenter de ramener une âme sur terre, le premier à vouloir vaincre la mort et à revenir de la mort. Le pouvoir surnaturel de sa musique est pareillement duel: il calme les tempêtes lors de l'expédition des Argonautes mais, en même temps, il fait taire les sirènes, leur intimant ainsi leur mort prochaine ${ }^{6}$.

Orfi, dans Poema a fumetti, est une pop star. Lui séduit les filles dans les boîtes de nuit (p.38-39), fait céder la porte de l'enfer et son gardien (p.198), enthousiasme la foule des âmes qui peuple ces espaces funèbres (p. 154-155). Le contenu des chansons d'Orphée n'est connu, dans la version ovidienne, qu'au retour de l'Hadès pour justifier la fin tragique du chantre. Buzzati choisit de les révéler au fur et à mesure du récit. Comme un leitmotiv, les ritournelles d'Orfi parlent d'amour (p. 180-181), de dieu (p. 192-193), d'angoisse (p. 133-135) et de mort. La mort hante littéralement le chant (La Storia dell'uomo che si voltò, p. 164; La Storia dei nove gentiluomini, p. 166; La Storia del casellnate, p. 168). La révélation des paroles explique l'effet qui en découle, la porte qui s'ouvre et l'autorisation de partir à la recherche de la femme aimée et défunte ${ }^{7}$.

Quel que soit le public, vivant ou mort, les chansons accompagnées de leur mélodie subjuguent. Le pouvoir de séduction de la musique d'Orfi passe par les mots et les souvenirs qu'ils éveillent. Immanquablement, la douce violence de la nostalgie capture les auditeurs. Elle touche leurs désirs les plus secrets. La mélancolie d'un temps révolu agit comme un charme. De là découlent la soumission des volontés, le désir impérieux d'écouter en se remémorant ce qui disparaîtra ou ce qui a déjà, à jamais disparu. La séduction de ce chant - la survivance qui s'y joue - touche au plus intime l'être de celui qui l'entend. Buzzati suggère ainsi le pouvoir du chant de ramener à la vie, de faire renaître.

Les images que dessine Buzzati sollicitent à leur tour l'attention. Par leur contenu et leur plasticité, elles captivent. Elles montrent la séduction à l'œuvre et parallèlement font œuvre de séduction. La séduction circule.

6. Les Argonautiques orphiques, Paris, les Belles Lettres, 1987, 1284-1297: "Tandis que je jouais de ma cithare, les Sirènes, depuis la cime de leur guette, furent prises de stupeur et mirent fin à leur chant. De leurs mains, elles lâchèrent, l'une les roseaux de la flûte et l'autre sa lyre. Elles poussèrent d'affreux gémissements, car le triste jour de leur mort, fixé par le destin, était arrivé».

7. «Sì, ragazzo Orfi, ci hai abbastanza emozionati. SEI GIOVANE, MA HAI GIÀ CAPITO MOLTE COSE. Puoi passare. Puoi entrare. Ti sono concesse ventiquattro ore» (Poema a fumetti, p. 198). 
Elle passe du récit au lecteur/regardeur. Mais pour en saisir le fonctionnement, il faut d'abord, se laisser gagner par le pouvoir hypnotique de l'image.

\section{Les images du féminin}

\section{La disponibilité des femmes}

Milan permet à Buzzati d'évoquer l'univers infernal. À l'image de la ville s'ajoute celle du bordel. On y pénètre dès le seuil de la première porte. Une femme en petite tenue, guêpière, jarretelles, bas sombres et escarpins à talon, accueille Orfi et l'invite à entrer (p.72-80). Descendant plus avant, il croise d'autres filles toutes aussi aguicheuses et dévêtues que la première. Le pays des morts est peuplé de femmes impudiques et lascives. Leur nombre suscite la fascination et renvoie le regardeur à son désir tandis que leur érotisme branche directement la séduction sur leur corps, les magnétise comme réservoir de flux. Ces corps, servis par un dessin stéréotypé, se commuent en force active qui happe. La stratégie est sommaire et imparable: elle se nomme exhibition.

Dessinées en plan rapproché sur une seule page, les filles découvrent leurs seins ronds aux tétons roses et pointus, leurs fesses charnues, leurs tailles fines et souples. Elles jouent l'innocence tout en invitant à la licence. En s'amusant avec leur chevelure ondoyante (p.206), en aguichant par des regards envoûtants et directs (p.114-115, 117), elles miment la proposition sexuelle. Leurs poses à la limite de la vulgarité - des hanches cambrées (p. 116, 210), des jambes écartées (p. 113), des bouches pulpeuses et entrouvertes, un doigt entre les lèvres (p. 107, 211)... tous les stéréotypes de l'érotisme rebattu et facile ici répertoriés - suggèrent l'obscénité. Une ligne noire et régulière contourne chacun des corps féminins, quasiment tous de la même teinte chair, sans nuance, sans ombre et lumière. Ainsi dévoilés et fétichisés, ils accèdent à un statut de signes. Au fil des pages, la chartre graphique crée une unité visuelle, introduit un rythme répétitif où la provocation de la nudité prédomine. Celle-ci sature l'ensemble du livre. Image envahissante, elle soutient l'attention, attire les regards. Invitation perverse, elle éveille le désir de voir toujours plus.

Les dessins témoignent de l'image d'un féminin affriolant et tentateur, tout entier adressé aux fantasmes masculins. Tous ces corps réduits à un seul, à chaque fois identique, ne proposent qu'une seule valeur d'usage, la disponibilité sexuelle. L'image de celle qui prend l'initiative - la séductrice 
- n'est concevable, ici, que sur le mode économique de la prostitution, qu'enfermée dans l'espace clos du bordel, réduite à être un objet de plaisir. Or, en même temps que son corps s'expose, il fait advenir l'image d'un autre corps, l'image spectrale et non moins fantasmatique d'un corps perdu, son double et son antithèse, pur et intouchable.

\section{Éros et Thanatos}

Les images luxurieuses sont d'autant plus envahissantes qu'elle sont relayées au cours du récit par les thèmes des chansons d'Orfi. Le premier chant, Le Streghe della città (p. 40-47) met en scène des prostituées. La seconde mélodie, Il Visitatore del pomeriggio (p. 172-175) raconte le cauchemar d'une dormeuse attaquée et totalement pénétrée jusqu'au trépas, par un monstre lancéolé et arachnéen. L'action de la mort se superpose à la licence des représentations. Elle se glisse entre ces dessins sulfureux. À chaque fois de façon différente - un pendu (p. 30), des crânes (p. 34, 147, 151), des cimetières (p.140,193), des visages ou des corps inanimés (p.61-62, 233), un oiseau de mort (p.67) ou un cortège funéraire (p. 57$58)$-, elle trouble et inquiète l'image.

La mort est sans relâche à l'œuvre dans le livre de Buzzati. Or, la force noire de la séduction dérive de l'intrication de l'érotisme avec la mort. La référence à Georges Bataille ne dit rien d'autre. L'allusion textuelle à «la petite mort» (p. 191) est annoncée tout d'abord par un dessin (p.103) où un crâne, dissimulé dans l'enchevêtrement de corps sensuels sourit, indiquant la vanité de la chair et du plaisir, puis par la reprise d'une photographie de la célèbre strip-teaseuse, Mademoiselle Féline (p.109) ${ }^{8}$, qui cachait son anatomie sous deux têtes de mort grimaçantes. L'érotisme incarne cette capacité à succomber, à mourir suavement. L'arrachement au monde par le ravissement dessine la béance dans laquelle se glisse le dernier souffle de l'agonie ou de l'orgasme. Par cet effondrement des corps et par l'ébranlement de l'être, les repères disparaissent et les limites sont transgressées, dépassées pour atteindre le vertige du désir satisfait.

La troisième figure de la séduction vient déjouer le risque d'une atmosphère lugubre et mortifere. En effet, la citation contrebalance les effets de la pulsion de mort par l'abondance des références, la malice des allusions, la ruse des évocations; citation qui est ici, l'outil principal de la création plastique buzzatienne.

8. Chevalier D., Métaphysique du strip-tease, Paris, Pauvert, 1961. 


\section{La fabrique des images: la citation comme figure de la séduction}

\section{Citer}

Les dessins de Poema a fumetti sont majoritairement des citations: citations de reproductions issues d'un large fonds culturel, de photographies réalisées sous la direction de Buzzati ${ }^{9}$ et de reprises de ses peintures antérieures. La citation pénètre l'essentiel de l'œuvre sous des modalités plurielles, par accumulations stratifiées prenant la forme du palimpseste, par plagiat ou par allusion. Poema a fumetti est, dans son ensemble, sous l'influence du Pop Art américain que Buzzati connaît bien car il a rencontré, lors d'un voyage à New York, en 1965, les principaux acteurs de la pop culture. Buzzati leur emprunte leurs procédés techniques (peinture acrylique, usage de la photographie et du collage) et l'introduction de la culture populaire dans l'univers des beaux-arts.

De Wesselmann, Buzzati retient surtout son esthétique de l'impudeur et reprend à son compte l'indécence des nus allongés, la provocation des poses où les corps s'étirent, les jambes s'écartent, les langues se tirent. Cette peinture rutilante offre une image stéréotypée de la lascivité où, pour une convergence de significations, lit, fourrure, poils, peau, lèvres, cigarette incandescente, fruits ou fleurs fétichisent la sexualité.

Les peintures de Wesselmann sont directement inspirées de la culture de masse. Les pin up des dépliants publicitaires ont souvent servi de point de départ à ses compositions. Le peintre renouvelle leur aspect glamour pour en souligner le sex-appeal. Mais plus que le surgissement de nudités obscènes produites durant les années pop, le thème dominant est bien plutôt l'exhibition de quelques perversions sexuelles, fétichisme et sadomasochisme par exemple.

L'enfer de Poema a fumetti est un bordel. À la manière des filles peintes par Richard Lindner ou par Allen Jones, qui privilégient l'aspect tactile des textures (peau, tissus, cuir, latex ou fourrure) et qui développent ainsi le spectacle de l'ultra-féminin soit dominateur, soit totalement dominé, les petites putes en déshabillé que croise Orfi participent de cette esthé-

9. Dino Buzzati réunit en 1968, les principaux acteurs de Poema a fumetti et les photographie à la manière des romans-photos. Il fixe les mimiques nécessaires pour ses futurs dessins. Une fois développées, les photographies sont recopiées ou décalquées. Voir Buzzati 1969 : il laboratorio di "Poema a fumetti", Milan, Mazzota, 2002, p. 72. 
tique. Buzzati reprend les codes fétichistes, s'attache aux corps et aux vêtements et accroît ainsi la charge érotique du livre.

Même si les évocations de pratiques sadomasochistes sont discrètes dans Poema a fumetti, Buzzati, dans ses remerciements, reconnaît sa dette envers l'éditeur et photographe new-yorkais, Irving Klaw, grand initiateur de la photographie bondage avec son modèle culte, Betty Page, et promoteur actif de nombreuses bandes dessinées sadomasochistes comme celles d'Éric Stanton ou d'Eneg, sources d'inspiration de quelques dessins de Buzzati ${ }^{10}$.

Le recyclage des images se poursuit. La citation relève du principe du découpage et du collage. Elle est prélèvement et appropriation de fragments élus. Poema a fumetti est un réseau de citations et se rapproche en cela du travail de Rauschenberg. Par ses sérigraphies ou ses Combine Paintings, Rauschenberg dépersonnalise l'acte de peindre en choisissant des photographies, des coupures de journaux, des lettres, de la bande dessinée, des images préfabriquées. Il fait se rencontrer des éléments hétéroclites employés librement, combinés de façon constructive ou placés dans des rapports aléatoires, donnant le sentiment d'une absence d'interprétation à cet ensemble désordonné, morcelé et touffu. Malgré des processus de création mécaniques qui désindividualisent et objectivent les images, Rauschenberg arrive à une œuvre chargée de significations intimes. Les citations choisies par Buzzati pour Poema a fumetti, sans être aussi prolixes et complexes que celles de l'Américain, fonctionnent à l'identique. Buzzati emprunte des images au très sérieux Atlante di medicina legale de Prokop et Weimann (p.30), à la bande dessinée (la reine de Sogo dans Barbarella de Jean-Claude Forest ${ }^{11}$, le personnage à nez d'oiseau dans Batman, la tête de mort de la couverture d'un numéro de Kriminal ${ }^{12}$ [p.34] et certaines mises en page de Guido Crepax ${ }^{13}$ pour Valentina [p. 108, 188 et 238]), à l'illustration (une aquarelle pour Rip van Winckle d'Arthur Rackham ${ }^{14}$ [p. 138], des dessins d'Otto Greiner [p. 139] ou d'Achille Beltrame [p.170], tous deux illustrateurs pour des quotidiens

10. Poema a fumetti, p. 105 et Vicki, l'hérö̈ne de Éric Stanton dans Bound in leather, 1953; Poema a fumetti, p. 141 et la position en arc d'Elexus, héroïne d'Eneg, alias Gene Bilbrew dans Island of captive, 1953.

11. Forest J.-Cl., Barbarella, Paris, le Terrain vague, 1964.

12. Batman, $\mathrm{n}^{\circ} 36,23$ giugno 1968, Milano, A. Mondadori Editore; Kriminal, ${ }^{\circ}{ }^{5}$, dicembre 1964, couverture de Luigi Corteggi, Milan, Editoriale Corno.

13. Crepax G., Valentina, Milan, Libri edizioni, 1968.

14. Rackham A., Les Monts Kaatskills ont toujours été hantés, 1904. Plume et aquarelle, 39 x 30, localisation inconnue; illustration extraite de Rip Van Winkle (\$7) de Washington Irving, 1905. 
milanais). Il cite un photogramme de Nosferatu le vampire de Murnau (p. 150), des photographies de revues érotiques (p. 41, 43, 172). Il réemploie sa propre peinture (Urlo, Santa ingenuità, Il visitatore del mattino, entre autres) ${ }^{15}$. En revanche, Buzzati ne convoque que des impressions provenant des univers esthétiques de Dalì ${ }^{16}$ (la déliquescence des formes [p.51-52,163]), de Friedrich ${ }^{17}$ (l'immensité des espaces vides [p. 130]) ou de Bellmer ${ }^{18}$ (l'érotisme polymorphe des corps et la linéarité du graphisme [p. 183]). Buzzati dessine ensuite ce qu'il connait, Milan, ses rues (p.35) et ses monuments, la tour Velasca (p.45), la tour Pirelli (p. 89), le dôme (p.194, 204-205). Il représente ses amis, le peintre Antonio Recalcati (Orfi), le journaliste Franco Gremignani (l'Homme en vert), Runa Pfeiffer (la Servante des enfers), Almerina, son épouse (Eura). Les illustrations de Buzzati suggèrent encore d'autres références, celles-là implicites: les calligrammes d'Apollinaire (p.110), la lithographie d'Arachnée pour Le Purgatoire de Dante de Gustave Doré (p. 175), le portrait photographique tremblé, au regard dédoublé de la Marquise Casati par Man $\operatorname{Ray}^{19}$ (p.49), les différentes visions de la gare de Milan de Pasolini et de Fellini ${ }^{20}$ (p.212-215), les tableaux expressionnistes, La Madone (p. 206) et Le Cri (p. 126) de Munch ${ }^{21}$ ou encore les espaces urbains désertés des peintures de De Chirico et de Sironi ${ }^{22}$ (p. 70-71, 99,

15. Buzzati D., Urlo, 1967. Acrylique sur papier, 30 x 39, localisation inconnue; Poema a fumetti, p. 33. Santa ingenuità, 1966. Acrylique sur toile, 100 x 65, coll. privée; Poema a fumetti, p. 49. Il Visitatore del mattino, 1963. Technique et localisation inconnues; Poema a fumetti, p. 173-175.

16. Dalì S., Le Téléphone/Homard, 1936. Assemblage d'objets, 18 x 12 x 30, Edward James Foundation, en prêt à Rotterdam, Museum Boymans-van Beuningen; L'Énigme de Hitler, 1939. HT, 95 x 141, Madrid, Museo Nacional Centro de Arte Reina Sofia.

17. Friedrich C.D., Abbaye dans un bois de chênes, vers 1809. HT, 110,40 x 171, Berlin, Nationalgalerie; Collines et champs près de Dresde, 1824. Huile sur bois, 22,20 x 30,50, Hambourg, Kunsthalle.

18. Bellmer H., Sans titre, 1962. Crayon à papier sur feuille quadrillée, 16,50 x 18, coll. privée; Sans titre, 1965. Crayon à papier, 50 x 59, coll. privée.

19. Ray M., La Marquise Casati, 1922. Positif argentique sur plaque de verre, 24 x 18, Paris, Musée national d'art moderne.

20. Pasolini P.P., Teorema, 1968. Le cadrage des dessins de Buzzati ressemble au panoramique cinématographique sur la verrière et la trouée de lumière qui se déplace ensuite vers la droite, resserrant l'image sur les trains, à la fin du film. Federico Fellini avec lequel Buzzati collabore en 1965 pour le scénario du film jamais réalisé, Il Viaggio di G. Mastorna, et avec lequel il invente cette image de trains à étages ressemblant à des villes sur rail.

21. Munch E., La Madone, 1893. HT, 90 x 68, Oslo, Munch-Museet; Le Cri, 1893. Huile, tempera et pastel sur carton, 84 x 67, Oslo, Munch-Museet.

22. De Chirico G., Méditation automnale, 1912. HT, 55 x 70, coll. privée; Gare Montparnasse (La Mélancolie du départ), 1914. HT, 140 x 184,50, New York, Museum of Modern Art.. Sironi M., Periferia (il tranvia e la gru), 1920. Huile sur papier entoilée, $98 \times 72$, Milan, coll. privée; Compositione de architettonica urbana, 1923. HT, 58 x 80, Vérone, Galleria dello Scudo. 
131). Buzzati prélève, mélange et recompose pour élaborer Poema a fumetti. Il recycle une masse considérable de documents. Ce recueil est fabriqué à partir de fragments d'images, de morceaux de peintures, de souvenirs de représentations, de lieux familiers, d'êtres proches. De la même manière que pour Rauschenberg, l'accumulation lui permet d'aboutir à une polarité entre objectif et subjectif.

De Lichtenstein et de Warhol, Buzzati retient l'introduction des codes de la bande dessinée dans la peinture ${ }^{23}$. Dans Poema a fumetti, une majorité de pages est divisée en cases. Les phylactères sont écrits en majuscules. Un contour noir et régulier détoure tous les dessins. La couleur est sobre et uniforme. Des bulles de dialogue, de pensée, des onomatopées animent le texte. Alors que pour les deux pop artistes, l'agrandissement d'une case était un moyen de vider de sa symbolique la peinture et d'éliminer le message narratif de la bande dessinée, pour Buzzati, le recours à la bande dessinée permet d'établir un pont entre dessin et écriture et de faire cohabiter les deux modes d'expression.

Le Pop Art, en choisissant comme ligne directrice la répétition, l'anonymat mécanique, la récupération des images toutes faites, questionne l'originalité et la paternité de l'œuvre ainsi que les rapports entre culture savante et culture de masse. La composition de Poema a fumetti ne progresse pas autrement. Le recours extrêmement riche à la citation fonctionne comme un montage signifiant. Les images sont issues de réalités extrêmement éloignées les unes des autres pour être combinées entre elles. Poema a fumetti sonde les interactions de la citation au sein de l'œuvre et ses répercussions sur les deux messages, linguistique ${ }^{24}$ et iconique.

\section{Citer et séduire}

La citation est appropriation. Elle recycle des choses toutes faites. En pénétrant l'image, elle se modifie et la modifie. Il y a les mêmes œuvres et l'œuvre même. En faisant jouer les images les unes avec les autres, la citation fait éclater l'objet de départ pour lui proposer une autre destination, une nouvelle singularité. Le rapport n'est pas d'équivalence ni de redondance car la citation creuse le texte et l'image. Elle les ouvre, elle les écarte pour un départ vers de nouvelles significations. Il y a, dans son utilisation,

23. Lichtenstein R., Drowning Girl, 1963, HT, 172,5 x 172,5, New York, Museum of Modern Art; Andy Warhol, Superman, 1960, Acrylique sur toile, 170 x 133, coll. G. Such.

24. Le texte recèle de multiples références, il faudrait penser à Ovide, Dante, Lewis Carroll, Apollinaire, Tennessee Williams, sans oublier les nouvelles et romans de Buzzati. 
une volonté de détourner le sens premier. Sa force est justement son installation dans l'écart, écart entre l'original et la reprise, écart d'autant plus grand que Buzzati ne fait pas de citation littérale et manipule que des fragments.

La citation agit comme un déclencheur, comme le moteur d'un travail de création dans l'imaginaire. Elle amorce l'élaboration de la lecture. Elle fait appel au lecteur/regardeur et à sa capacité à recevoir, à reconnaitre et à s'emparer de sa proposition pour l'appliquer à autre chose. Donc, par un phénomène de remémoration, la citation suscite l'interrogation. Elle éveille la curiosité; les multiples références de Buzzati surprennent. C'est justement dans cet étonnement que s'installent conjointement son pouvoir mobilisateur et la parade du séducteur.

La séduction est une force qui machine, esquisse, aguiche, se dérobe, s'avance jusqu'à devenir insaisissable. Les jeux de citations dans Poema a fumetti sont intrigants à plus d'un titre: ils mettent en scène des images érotiques qui fétichisent le corps féminin cantonné à un état d'objet sexuel ou d'objet à asservir et à punir, punir d'avoir été désiré; ensuite, ils misent sur le pouvoir de fascination de la mort en montrant ce qui se passe dans l'au-delà; enfin, le recours à la citation joue sur les différents niveaux de la répétition où chaque référence s'enchaîne, se rapproche et en entraîne une nouvelle, dilatant sans fin le champ de la reconnaissance. La citation buzzatienne entretient avec la mémoire une liaison exubérante: elle semble former la circularité ininterrompue de l'anneau de Möbius car l'identification n'arrête pas de se modifier, de se moduler, d'osciller pour finir par tanguer. Dans l'ambiguïté se joue l'action de la séduction.

Les citations sont comme des gestes furtifs à l'encontre du regardeur, des coups d'œil facétieux. Elles sont en quelque sorte une métaphore de l'amour. Elles sont l'équivalent de la cristallisation qui travaille le coup de foudre. Elles décomposent l'image envoûtante mais pour la recomposer aussitôt, la cadrer, la condenser. Dans la peinture tout comme dans l'écriture de Buzzati, la citation tente de reproduire une passion de lecture et d'amateur d'art, de retrouver l'instantanée fulgurance de la sollicitation originelle. La citation répète, elle fait retentir la lecture dans l'écriture, le regard dans la peinture. Par là même, elle invite et sollicite; elle joue sur le désir du regardeur. Elle est un ébranlement, un ravissement avant même sa compréhension tandis que l'excitation est à la recherche dans l'image, de la cause de cette sollicitation.

À la manière du séducteur qui engage une mise en scène précieuse et calculée pour attirer et retenir, la citation, tout en transportant du sens, emprunte des correspondances pour aller ailleurs. Elle nous convoque et 
nous déplace vers une série infinie. Citare en latin signifie mettre en mouvement, faire passer du repos à l'action. Les sens des verbes faire venir à soi, appeler mais aussi provoquer entrent dans le champ lexical de la citation et de la séduction. Une même puissance est en jeu, celle qui met en branle. Dans le vocabulaire de la corrida, on dit que le torero "cite" le taureau, c'est-à-dire qu'il provoque sa charge à distance, le promeut en agitant un leurre devant ses yeux. "C'est certainement cet emploi qui demeure le plus fidèle au sens premier et essentiel de la citation" constate Antoine Compagnon ${ }^{25}$; c'est aussi le sens de la séduction. La séduction n'est ni plus ni moins qu'un leurre vers lequel se dirige l'être séduit. Tout comme la citation, elle entraîne et déplace. Et dans les deux cas, cela suppose de l'adhésion; cela nécessite d'être vulnérable, en instance d'être séduit; c'est se mettre en état d'être affecté, devenir poreux, s'ouvrir, s'abandonner, se perdre. Si, dans la séduction, il y a une forme de rapt, il y a nécessairement alors oubli de soi. Séduire, c'est donner confiance à l'autre et l'obtenir pour induire un mouvement en retour et ainsi obtenir que ce dernier revienne du soi de l'autre. Mais pour cela il faudra que le séducteur ait agi et touché.

\section{Le séducteur buzzatien}

Le séducteur est un professionnel, un personnage dangereux qui a une idée derrière la tête, une stratégie, des moyens, des objectifs qu'il dissimule. Il dispose du séduisant pour arriver à ses fins et, notamment, il sait ce qu'il en est du désir de l'autre. À quelle catégorie appartient notre séducteur littéraire? Plutôt débauché ou plutôt libertin?

Le libertin au XVIII ${ }^{e}$ siècle est celui qui trompe, abuse de la crédulité de sa victime pour l'abandonner ensuite. Il est le cynique collectionneur, le trompeur destructeur tandis que le débauché est celui qui reconnaît la validité du code qu'il enfreint, la légitimité des règles qu'il transgresse et auxquelles il se soustrait parce qu'il a le sentiment de la légèreté de sa faute, de l'absence d'intention malveillante à l'encontre de l'autre, parce qu'il y a consentement mutuel. L'autre est le partenaire et non la victime ou l'objet passif.

Avec Poema a fumetti, Buzzati tire les ficelles, se joue des œuvres pour les recycler. Au moyen des citations, il manipule son lecteur et manipule la

25. Compagnon A., La Seconde main ou le travail de la citation, Paris, Seuil, 1979. 
littérature, une littérature qui traite de la séduction mais, plus sûrement, une littérature qui a pour but la séduction. Le texte de Buzzati dit et ne dit pas ce qu'il est. Ses images citent et ne citent pas. Cette démarche est à rapprocher du sens originel de séduire, mener à l'écart et détourner. Buzzati nous ensorcelle, nous attire dans son réseau de références. Il nous détourne du sens du récit pour nous conduire sur d'autres voies en titillant notre désir de voir, de connaître et d'identifier. Il nous charme. En réalité, il est Orphée. Mais Orphée n'est-il pas l'archétype de l'artiste, et son mythe le symbole de la création? Que cherche à nous donner Buzzati, si ce n'est l'origine même de l'œuvre, dans sa très grande fragilité parce qu'il sait qu'à tout moment, elle peut retourner dans la nuit. «Orphée ne chantait pas, il peignait " écrit Jean-Luc Marion ${ }^{26}$, et le peintre, ici, a le pouvoir de rendre visible ou plutôt de faire monter l'invisible. 